INPLASY

PROTOCOL

To cite: Wang et al. The longterm effects of delayed cord clamping on infants: A system review and meta analysis. Inplasy protocol 202130046. doi:

10.37766/inplasy2021.3.0046

Received: 15 March 2021

Published: 15 March 2021

Corresponding author:

Limin Wang

1501728992@qq.com

Author Affiliation:

Union Hospital, Tongji Medical

College, Huazhong University

of Science and Technology

Support: No.

Review Stage at time of this submission: Preliminary

searches.

Conflicts of interest:

None declared.

\section{The long-term effects of delayed cord clamping on infants: A system review and meta analysis}

\author{
Wang, L1; Lu, C2; Hu, M33; Xiong, S4; Wang, M5.
}

Review question / Objective: Compared with early umbilical cord clamping, whether delayed umbilical cord clamping can bring more benefits to the baby in the long run.

Condition being studied: A number of studies have shown that delayed umbilical cord clamping can improve hematology, reduce the risk of necrotizing enterocolitis, and reduce the chance of intraventricular hemorrhage, especially within one week after birth. In addition, some researchers have suggested that delayed cord clamping increases the risk of polycythemia, the need for phototherapy, and transient shortness of breath in newborns. In the first 6 months of life, the delayed clamping that leads to increased blood circulation in the newborn may be harmful and may lead to an overload of the newborn's blood volume. Therefore, it is necessary to systematically review the effectiveness of delayed cord clamping.

INPLASY registration number: This protocol was registered with the International Platform of Registered Systematic Review and Meta-Analysis Protocols (INPLASY) on 15 March 2021 and was last updated on 15 March 2021 (registration number INPLASY202130046).

\section{INTRODUCTION}

Review question / Objective: Compared with early umbilical cord clamping, whether delayed umbilical cord clamping can bring more benefits to the baby in the long run.
Condition being studied: A number of studies have shown that delayed umbilical cord clamping can improve hematology, reduce the risk of necrotizing enterocolitis, and reduce the chance of intraventricular hemorrhage, especially within one week after birth. In addition, some researchers 
have suggested that delayed cord clamping increases the risk of polycythemia, the need for phototherapy, and transient shortness of breath in newborns. In the first 6 months of life, the delayed clamping that leads to increased blood circulation in the newborn may be harmful and may lead to an overload of the newborn's blood volume. Therefore, it is necessary to systematically review the effectiveness of delayed cord clamping.

\section{METHODS}

\section{Participant or population: Newborn baby.}

Intervention: Delayed cord clamp: after the umbilical cord stops beating, or clamps after the placenta is separated, or clamps the thread more than 60 s after delivery.

Comparator: Immediate umbilical cord clamp: clamp within 60 s after delivery.

Study designs to be included: Randomized controlled trials.

Eligibility criteria: If the study is a randomized controlled clinical trial of neonates that compares immediate cord clamping with delayed cord clamping, and the follow-up time exceeds one month, these studies are considered eligible for inclusion. At the same time, we define early clamping as clamping within 60 s after delivery, and define delayed clamping as clamping after the umbilical cord stops beating, or after the placenta is separated, or more than 60s after delivery. The exclusion criteria are as follows: observational studies and retrospective studies, narrative and systematic comments, case reports, letters to the editor, and editorials.

Information sources: We will use selected keywords and MeSH terms to conduct systematic searches in Pubmed, EMBASE and Cochrane libraries. In addition, we will also screen the references of key documents included in the study.
Main outcome(s): Serum ferritin, anemia, iron deficiency, AAQ--(監), hemoglobin and so on.

Quality assessment / Risk of bias analysis: Two researchers independently evaluated the risk of bias based on the Cochrane Collaborative Bias Risk Tool. Controversial articles and items were invited to evaluate a third researcher and discuss the results.

Strategy of data synthesis: For binary variables, choose RR/OR. Select SMD/MD for continuous variables, and $H R$ for survival analysis data. When 12 is greater than $75 \%$, it shows significant heterogeneity. When it was determined that there was significant heterogeneity, we used the article-by-article exclusion method for sensitivity analysis.

Subgroup analysis: When $\mathbf{1 2}$ is greater than $50 \%$, it indicates significant heterogeneity. When it is determined that there is significant heterogeneity, we conduct a subgroup analysis based on the time the umbilical cord is clamped,

Sensitivity analysis: When $\mathbf{I 2}$ is greater than $50 \%$, it indicates significant heterogeneity, the article-by-article exclusion method will be used for sensitivity.

Country(ies) involved: China.

Keywords: Delayed cord clamp; Immediate umbilical cord clamp; Review.

Contributions of each author:

Author 1 - Limin Wang.

Author 2 - Caihong Lu.

Author 3 - Mengyun Hu.

Author 4 - Shasha Xiong.

Author 5 - Mingjing Wang. 